\title{
Carcinoembryonic antigen related cell adhesion molecule 6 promotes the proliferation and migration of renal cancer cells through the ERK/AKT signaling pathway
}

\author{
Rujian $\mathrm{Zhu}^{1,2 \#}$, Jiong $\mathrm{Ge}^{3 \#}$, Junjie $\mathrm{Ma}^{2}$, Junhua Zheng ${ }^{1,4}$ \\ ${ }^{1}$ Department of Urology, The Affiliated Shanghai No.10 People's Hospital, Nanjing Medical University, Shanghai 200072, China; ${ }^{2}$ Department \\ of Urology, Shanghai Pudong Hospital, Fudan University Pudong Medical Center, Shanghai 201399, China; ${ }^{3}$ Department of Radiology, Shanghai \\ Tenth People's Hospital, Tongji University School of Medicine, Shanghai 200072, China; ${ }^{4}$ Department of Urology, Shanghai General Hospital, \\ Shanghai Jiao Tong University School of Medicine, Shanghai 200080, China \\ Contributions: (I) Conception and design: R Zhu, J Ge; (II) Administrative support: J Zheng; (III) Provision of study material or patients: R Zhu, \\ J Ge, J Ma; (IV) Collection and assembly of data: R Zhu, J Ge, J Ma; (V) Data analysis and interpretation: R Zhu, J Ge; (VI) Manuscript writing: All \\ authors; (VII) Final approval of manuscript: All authors. \\ "These authors contributed equally to this work. \\ Correspondence to: Junhua Zheng. Department of Urology, The Affiliated Shanghai No.10 People's Hospital, Nanjing Medical University, Shanghai \\ 200072, China. Email: zhengjh0471@sina.com.cn.
}

Background: Carcinoembryonic antigen related cell adhesion molecule 6 (CEACAM6) is a versatile glycoprotein and a member of the CEACAM family. Studies suggested that it served as a diagnostic and prognostic biomarker in some malignancies. In addition, it is involved in tumorigenesis by stimulating proliferation, suppressing apoptosis, facilitating migration and invasion, promoting angiogenesis, and inducing drug resistance. In the present study, we demonstrated the oncogenic effects of CEACAM6 in clear cell renal cell carcinoma (ccRCC).

Methods: CEACAM6 expression was detected by quantitative real-time PCR (qRT-PCR), immunohistochemical staining and western blot in ccRCC tumor tissues and cell lines. Survival analysis was performed using the data of TCGA database. Cell proliferation and migration were detected by CCK8 and transwell assays with the overexpression or silencing of CEACAM6. LY294002 was used to block the activation of PI3K/AKT pathway. Associated pathway proteins were detected by western blot.

Results: CEACAM6 was upregulated in ccRCC cell lines and tumor tissues. Longer overall survival was observed in patients with relatively low CEACAM6 levels. Furthermore, overexpression of CEACAM6 promoted the proliferation and migration of ccRCC cells. Conversely, shRNA-mediated CEACAM6 depletion modulated those changes. Further investigation demonstrated that the ERK/AKT signaling pathway activation played a pivotal role. In addition, PI3K/AKT pathway blockade abrogated the effects of CEACAM6 overexpression.

Conclusions: Aberrantly high expression of CEACAM6 is a stimulus for the formation and progression of ccRCC.

Keywords: Carcinoembryonic antigen related cell adhesion molecule 6 (CEACAM6); clear cell renal cell carcinoma (ccRCC); proliferation; migration

Submitted May 12, 2019. Accepted for publication Aug 14, 2019.

doi: $10.21037 /$ tau.2019.09.02

View this article at: http://dx.doi.org/10.21037/tau.2019.09.02 


\section{Introduction}

Kidney cancers are common genitourinary cancers, more than $90 \%$ of which are renal cell carcinomas (RCCs) originating from the renal parenchyma (1). RCC is insensitive to conventional chemotherapy and radiotherapy (2). Therefore, surgical intervention, such as radical or partial resection of tumor-bearing kidneys, is the optimal treatment option for localized RCC (3). For metastatic RCC (mRCC), due to the expansion of therapeutic methods, resulting from the advent of targeted agents, systemic therapy using multi-kinase inhibitors, immunotherapies, surgery or a combination of them has been the mainstay of treatment (4-6). Although the management of RCC has been greatly improved in the past decade, many patients still suffer an unfavorable prognosis $(7,8)$. A better understanding of the pathogenesis and further exploration of novel biomarkers or therapeutic targets is therefore urgently needed.

Carcinoembryonic antigen related cell adhesion molecules (CEACAMs) comprise a multitude of glycoproteins belonging to the immunoglobulin superfamily. They are anchored to the cell surface though a transmembrane domain or glycosylphosphatidylinositol (GPI) anchor (9), and they mediate intercellular adhesion by homotypic or heterotypic binding with other CEACAM family members (10). Studies have demonstrated that CEACAMs are implicated in numerous cellular processes, such as cell adhesion, cell proliferation and angiogenesis, and tumorigenesis $(11,12)$.

As a versatile glycoprotein of the CEACAM family, extensive research suggests that CEACAM6 could serve as a biomarker for some human cancers. By detecting the CEACAM6 level, researchers found that high biliary CEACAM6 levels can predict the presence of an extrahepatic cholangiocarcinoma (13). In the current study on clinical samples, Upregulated levels of CEACAM6 indicate a poor prognosis for gastric carcinoma patients (14). In addition, CEACAM6 contributes to the development of some malignancies according to the clinical samples and cell culture experiments. For instance, CEACAM6 facilitates the proliferation of pancreatic carcinoma cells and promotes the metastasis of gastric cancers (15-17).

CEACAM family has not been extensively studied in RCC. Previous research indicates that CEACAM1 acts as a tumor suppressor gene in RCC (18). Ho et al. demonstrated the differential expression of CEACAM6 between patientmatched primary and mRCC (19). However, the role of CEACAM6 in RCC is still unclear. Hence, this study was designed to investigate the function of CEACAM6 in RCC.

\section{Methods}

\section{Tissue samples and cell culture}

clear cell (ccRCC) tissues $(\mathrm{n}=15)$ and adjacent normal kidney tissues ( $\mathrm{n}=15)$ were collected from ccRCC patients that underwent surgery in Shanghai Tenth People's Hospital after collecting informed consent and the approval of the Medical Ethics Committee. Human proximal tubule epithelial cell line HK-2 and ccRCC cell lines, containing 786-O, A498, OSRC-2 and SN12-PM6, were obtained from the American Type Culture Collection. All the cells grew in the media supplemented with $10 \%$ fetal bovine serum (FBS) and $1 \%$ Penicillin-Streptomycin at $37{ }^{\circ} \mathrm{C}$ in a humidified atmosphere containing 5\% $\mathrm{CO}_{2}$.

\section{$R N A$ isolation and quantitative real-time PCR (qRT-PCR)}

Total RNA was extracted from frozen tissues using Trizol reagent (Invitrogen, USA) according to the manufacturer's instructions. The concentration and purity of RNA was determined using an ND-2000 Spectrophotometer (Thermo Fisher Scientific, USA). Reverse transcription was performed using a PrimeScript RT reagent kit (TaKaRa, Japan), and qRT-PCR was performed with the KAPA SYBR FAST qPCR Kit (Kapa Biosystems, USA) using a 7900HT Fast Real-Time PCR System (Applied Biosystems, Japan). The CEACAM6 expression levels were normalized to $\beta$-actin. Data were analyzed using the $2^{\wedge}-\Delta \Delta$ Ct method. The primer sequences were as follows: CEACAM6 forward primer: 5'-TCAATGGGACGTTCCAGCAAT-3'; CEACAM6 reverse primer: 5'-CACTCCAATCGTGATGCCGA-3'. $\beta$-actin forward primer: 5'-GGGACCTGACTGACTACCTC-3'; $\beta$-actin reverse primer: 5'-TCATACTCCTGCTTGCTGAT-3'.

\section{Western blot analysis}

Cell samples were treated with RIPA lysis buffer (Beyotime Biotechnology) containing protease inhibitors to extract protein. After quantification with a BCA protein assay kit (Thermo Fisher Scientific), equal amounts of lysates were loaded into each polyacrylamide gel well. Proteins of interest were separated by electrophoresis and transferred onto nitrocellulose membranes. Then, the protein bands were blocked with $5 \%$ nonfat milk for $2 \mathrm{~h}$. Optimally 
diluted primary antibodies and corresponding secondary antibodies were used for immunoreaction. Finally, protein bands were scanned with an Odyssey infrared imaging system (LI-COR Biosciences). All the antibodies, including CEACAM6, AKT, p-AKT, ERK, p-ERK, C-MYC, MMP-9, Survivin, and $\beta$-actin were purchased from Abcam.

\section{Immunobistochemical staining}

Tissue samples were fixed with $10 \%$ formaldehyde and embedded in paraffin. Paraffin tissue blocks were sliced into sections. After deparaffinization with dimethylbenzene and rehydration with an ethanol gradient, endogenous peroxidase was inactivated by incubating with $3 \% \mathrm{H}_{2} \mathrm{O}_{2}$ for $10 \mathrm{~min}$. Then the sections were immersed in $10 \mathrm{mM}$ sodium citrate buffer solution ( $\mathrm{pH}$ 6.0) and boiled with a pressurecooker to retrieve the antigens. Bovine serum albumin was used for blocking non-specific binding sites. Afterward, the sections were incubated with a CEACAM6 antibody overnight at $4{ }^{\circ} \mathrm{C}$ and a corresponding secondary antibody for $40 \mathrm{~min}$ at $37^{\circ} \mathrm{C}$. Finally, the sections were stained with diaminobenzidine and counterstained with hematoxylin. Immunoreactivity was observed under a microscope.

\section{Plasmid construction and cell transfection}

CEACAM6 overexpression and silencing vectors were obtained from Genechem (China). To elevate CEACAM6 expression, GV230 plasmids were linearized by restriction endonucleases. The CEACAM6 (NM_002483) sequence was synthesized by PCR using the following primers. CEACAM6 (24388-1) Forward: TACCGGACTCAG ATCTCGAGCGCCACCATGGGACCCCCCTCAG CCCC; CEACAM6 (24388-1) Reverse: GATCCCGG GCCCGCGGTACCGTTATCAGAGCCACCCTGG CCAGCAC. Then the gene segments were integrated into linearized GV230 plasmids. PCR was performed to distinguish the bacterial colonies. The primers used were as follows. CMV-F: CGCAAATGGGCGGTAGGCGTG; pEGFP-N-3: CGTCGCCGTCCAGCTCGACCAG. The sequence of identified transformants were verified by gene sequencing. Similarly, sequences targeting CEACAM6 (GTATTGGTTACAGCTGGTA) were used to interfere with CEACAM6 expression. The recombinant plasmids or negative control vectors were transfected into RCC cell lines using Lipofectamine 2000 (Invitrogen).

\section{Cell Counting Kit-8 (CCK-8) assay}

A CCK-8 assay was conducted to assess cell viability. Briefly, 786-O or A498 cells were seeded into 6-well plates and subsequently transfected with recombinant plasmids to alter CEACAM6 expression. Then the cells were harvested and a $100 \mu \mathrm{L}$ cell suspension was added into each well of the 96-well plates. Five duplicates were set for each group. For rescue assays, the cells were additionally treated with the PI3K/AKT pathway inhibitor LY294002 (Selleck, s1105) for $24 \mathrm{~h}$. Finally, $10 \mu \mathrm{L}$ of CCK-8 was added to the culture medium after incubation. One hour later, the absorbance at $450 \mathrm{~nm}$ was measured using a microplate reader.

\section{Transwell assay}

Cell migration capability was assessed by transwell assay using transwell chambers (Corning, 3422). Similarly, 786-O or A498 cells were seeded into 6-well plates and transfected with GV230-CEACAM6, sh-CEACAM6, or negative control vectors. Forty-eight hours later, the cells were digested and resuspended with serum-free medium. A $200 \mu \mathrm{L}$ cell suspension was added into upper chamber. Fivehundred $\mu \mathrm{L}$ complete medium containing $10 \%$ FBS were added into the lower chamber. For the rescue assay, the cells were additionally treated with LY294002 for 24 h. Finally, the chambers were washed with PBS. Cells migrating to the lower surface of the polycarbonate membrane were treated with $4 \%$ paraformaldehyde and $0.1 \%$ crystal violet. Migrated cells from multiple fields of vision were counted to assess cell migration.

\section{Statistical analysis}

All the results were obtained from experiments conducted in triplicate. Data were expressed as mean \pm SD. Statistical analysis was performed with GraphPad Prism 6 (GraphPad Software, USA) using Student's $t$-test and one-way ANOVA. The correlation between CEACAM6 expression and overall survival of RCC patients was accessed by KaplanMeier analysis. Log-rank test was used to calculate p values. 

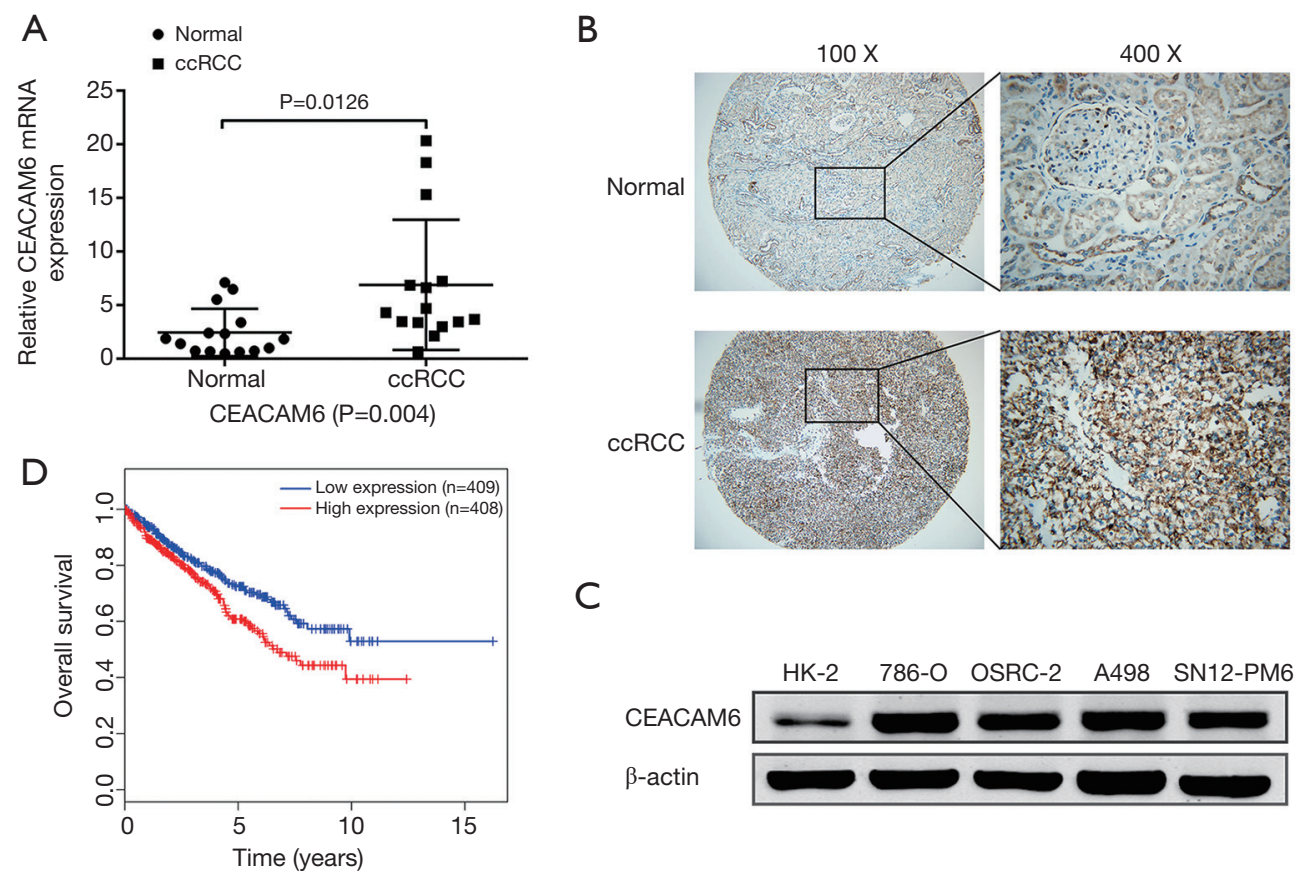

C

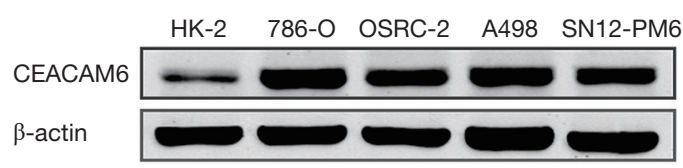

Figure 1 CEACAM6 was upregulated in both ccRCC tissues and cell lines and was associated with overall survival. ccRCC tumor tissues $(n=15)$ and normal kidney tissues $(n=15)$ were collected. (A) The relative mRNA levels of CEACAM6 were detected by qRT-PCR. P<0.05; (B) the increased expression of CEACAM6 was confirmed by immunohistochemical staining. The images were taken under a microscope with magnifications of $\times 100$ and $\times 400$; (C) the protein levels of CEACAM6 in several RCC cell lines and HK-2 cells were detected by Western blot; (D) Kaplan-Meier analysis of the correlation between CEACAM6 expression and overall survival for RCC patients was performed. $\mathrm{P}$ value was calculated by log-rank test. $\mathrm{P}<0.01$. CEACAM6, carcinoembryonic antigen related cell adhesion molecule 6; ccRCC, clear cell renal cell carcinoma; qRT-PCR, quantitative real-time PCR.

\section{$\mathrm{P}<0.05$ denoted statistical significance.}

\section{Results}

CEACAM6 was upregulated in both ccRCC tissues and cell lines and was associated with overall survival

Several studies have shown that CEACAM6 deregulation arises in a multitude of malignancies and it is related to tumorigenesis and metastasis. These features indicate the potential of CEACAM6 in serving as a novel biomarker or therapeutic target. Herein, we detected the differential expression of CEACAM6 in ccRCC tissues $(n=15)$ and normal kidney tissues $(n=15)$. According to the results of qRT-PCR, CEACAM6 mRNA levels were significantly elevated in RCC tissues $(\mathrm{P}=0.0126)$ (Figure 1A). Moreover, immunohistochemical staining further confirmed CEACAM6 was overexpressed in ccRCC tissues compared with normal tissues (Figure 1B). Subsequently, we detected the expression of CEACAM6 in ccRCC cell lines. In line with our other results, Western blotting revealed CEACAM6 protein levels were elevated in 786-O, OSRC-2, A498 and SN12-PM6 cells compared with HK-2 cells (Figure 1C). Accordingly, we can propose that the elevated level of CEACAM6 is a potential tumor promotor that is involved in the development of RCC.

In addition, the TCGA database was introduced to investigate the prognostic significance of CEACAM6 in RCC. The clinical data of 817 RCC patients was collected for Kaplan-Meier analysis. As shown in Figure 1D, patients with relatively high levels of CEACAM6 had shorter overall survival than those with low CEACAM6 expression, indicating a negative correlation between CEACAM6 expression and overall survival.

\section{CEACAM6 deregulation modulated the proliferation and migration of ccRCC cells}

To investigate the pathophysiological roles of CEACAM6 
A

786-O

A498

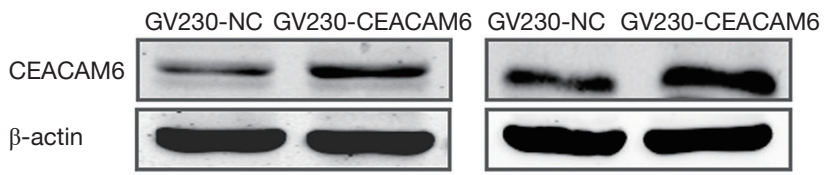

B

$786-0$

A498
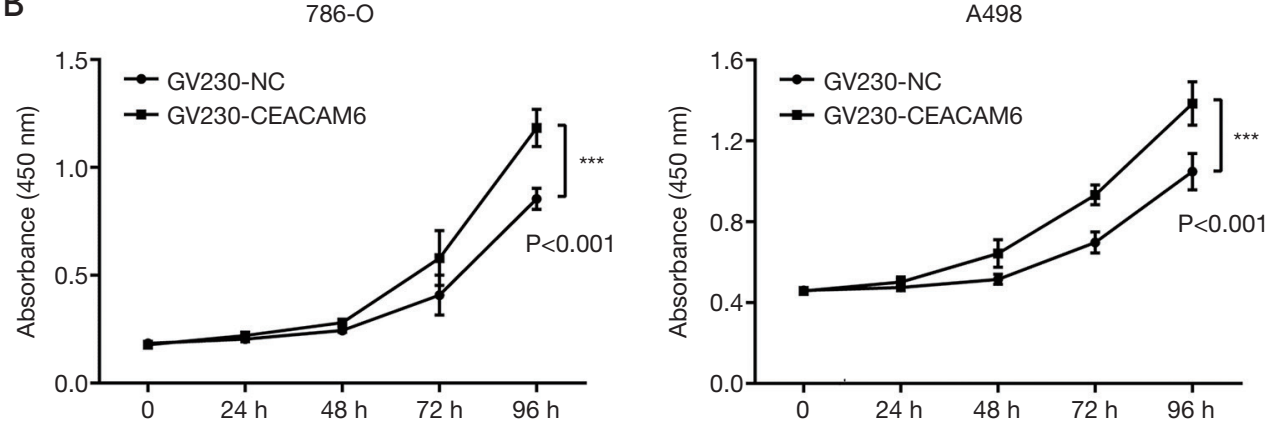

C GV230-NC GV230-CEACAM6
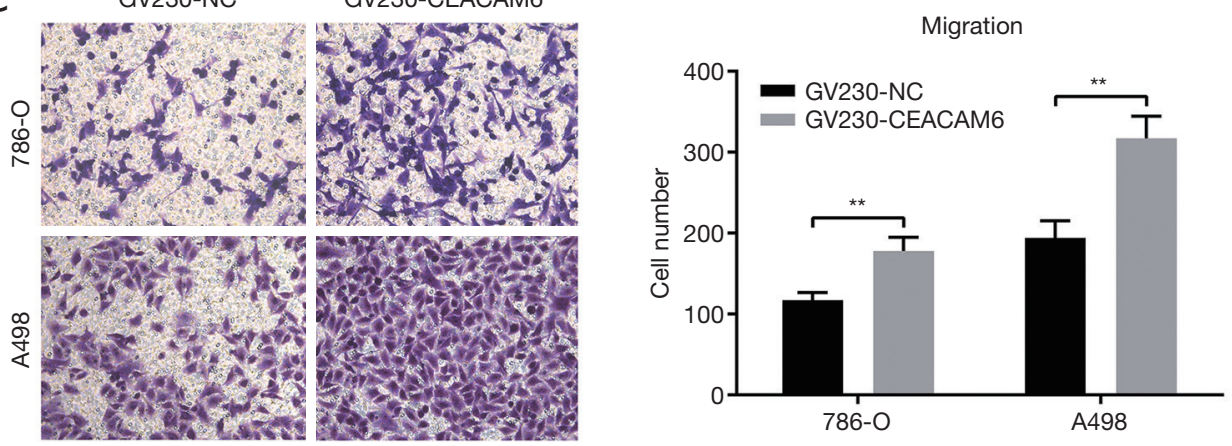

Figure 2 CEACAM6 overexpression facilitated the proliferation and migration of RCC cells. 786-O and A498 cells were transfected with GV230-CEACAM6 or GV230-NC (NC) to elevate CEACAM6 expression. (A) Transfection efficiency was confirmed by Western blot; (B) cell proliferation was detected by CCK-8 assay. The absorbances at $450 \mathrm{~nm}$ were examined after transfection for $0,24,48,72$, or $96 \mathrm{~h}$; (C) cell migration was detected by transwell assay. Cells were stained by crystal violet, $\times 200$. Data were analyzed by Student's $t$-test. **, $\mathrm{P}<0.01$; ***, $\mathrm{P}<0.001$. CEACAM6, carcinoembryonic antigen related cell adhesion molecule 6.

in ccRCC, recombinant plasmids containing CEACAM6 coding sequences were constructed to elevate the expression of CEACAM6 in 786-O and A498 cells. Transfection efficiency was confirmed by Western blot (Figure 2A). Then CCK- 8 assays were performed to assess cell viability. Increased CCK-8 was observed in CEACAM6 overexpressing ccRCC cells (Figure 2B), implying that CEACAM6 facilitates cell proliferation. In addition, cell migration was detected by transwell assays. CEACAM6 overexpression stimulated the migration of ccRCC cells (Figure 2C). In contrast, specific gene silencing vectors were used to knock-down the endogenous expression of CEACAM6. As shown in Figure 3A, targeting CEACAM6 with shRNA successfully interfered with the expression of
CEACAM6. Subsequent tests revealed that cell proliferation and migration were significantly suppressed following CEACAM6 silencing (Figure 3B,C).

In summary, CEACAM6 contributed to the growth and migration of ccRCC cell lines, which provided additional evidence that CEACAM6 is involved in carcinogenesis. Moreover, CEACAM6 silencing was effective in suppressing these malignant phenotypes.

\section{CEACAM6 exerted oncogenic effects in ccRCC through the ERK/AKT signaling patbway}

Subsequently, Western blotting was performed to clarify the underlying molecular mechanisms. It is well known 
A

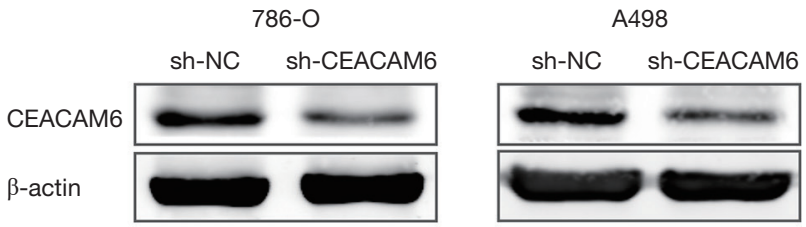

B

$786-\mathrm{O}$
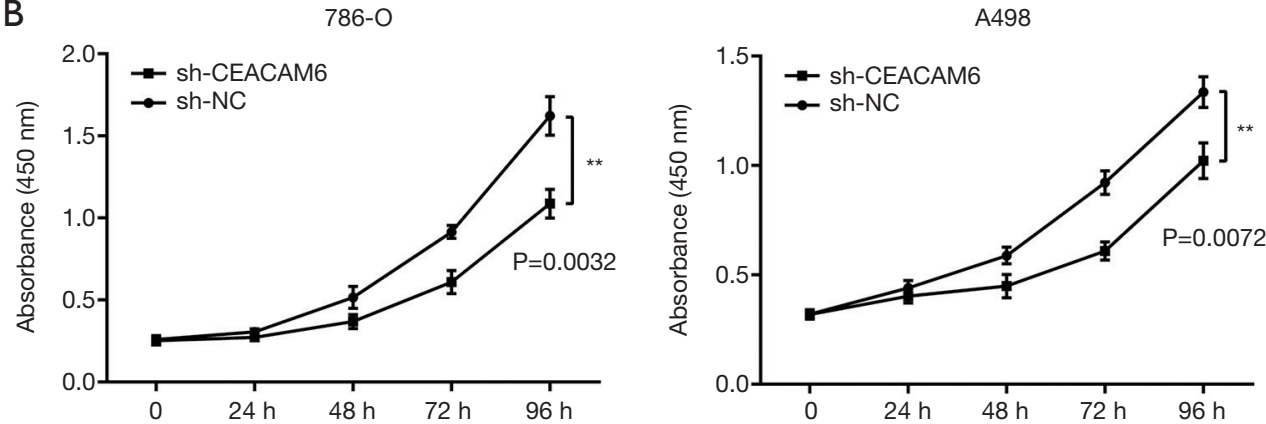

C
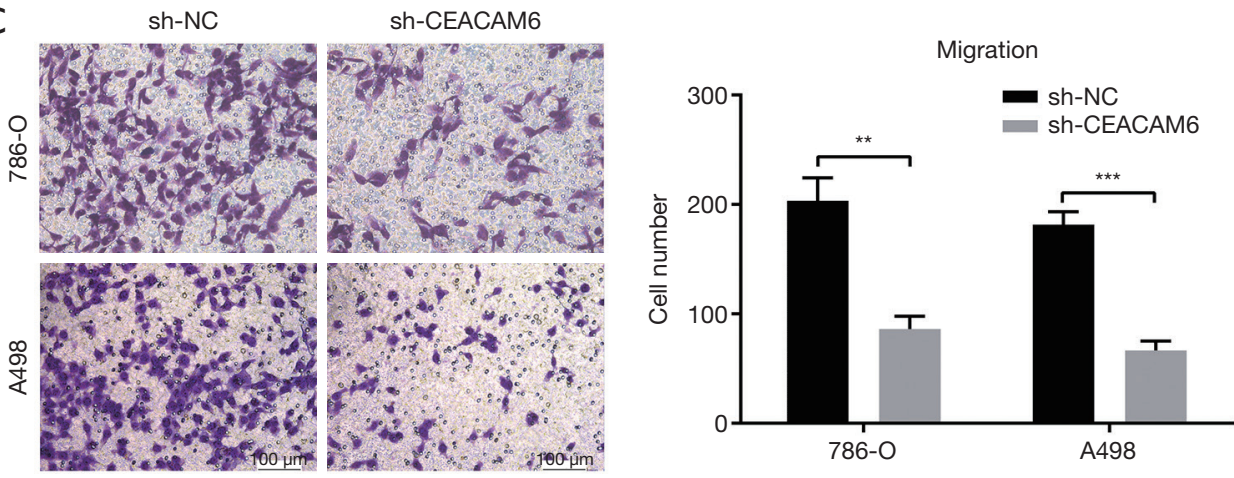

Figure 3 CEACAM6 silencing suppressed the proliferation and migration of RCC cells. CEACAM6-specific shRNAs were used to induce gene silencing in 786-O and A498 cells. (A) CEACAM6 silencing was confirmed by Western blot; (B) after transfection for 0, 24, 48, 72, or 96 h, cell viability was assessed by CCK-8 assay; (C) cell migration was detected by transwell assay, crystal violet staining, $\times 200$. Data were analyzed by Student's $t$-test. **, $\mathrm{P}<0.01$; ${ }^{* * *}, \mathrm{P}<0.001$. CEACAM6, carcinoembryonic antigen related cell adhesion molecule 6.

that ERK and AKT are pivotal signals for the initiation and progression of cancers by modulating downstream signaling cascades (20-22). After reducing CEACAM6 expression with specific shRNA, we found that phosphorylated ERK and AKT levels decreased, indicative of inactivation of the ERK/AKT signaling pathway (Figure 4A). Furthermore, $\mathrm{C}-\mathrm{MYC}$ and Survivin, two key oncogenes associated with cell growth and survival $(23,24)$, and migration elements, such as MMP-9, were also downregulated (Figure 4A). On the other hand, to investigate the activation of these signals by overexpressing CEACAM6, 786-O cells were transfected with GV230-CEACAM6 plasmids. Figure 4B demonstrated the activation of the ERK/AKT signaling pathway with the increased protein levels of p-AKT, p-ERK, C-MYC, Survivin, and MMP-9. To further verify this mechanism,
CEACAM6 overexpressing 786-O cells were treated with a specific PI3K/AKT pathway inhibitor. The capabilities of cell proliferation and migration were assessed by CCK- 8 and transwell assays. As shown in Figure 4C,D, increased cell proliferation and migration were abrogated by PI3K/AKT pathway blockage. The oncogenic effects of CEACAM6 were potentially ascribed to the activation of the ERK/AKT signaling pathway.

\section{Discussion}

CEA, also known as CEACAM5, has been identified as a colon cancer-associated antigen (25). CEACAM6, another GPI-anchored glycoprotein, like CEA, has attracted recent attention as another potential cancer-associated antigen. 
A

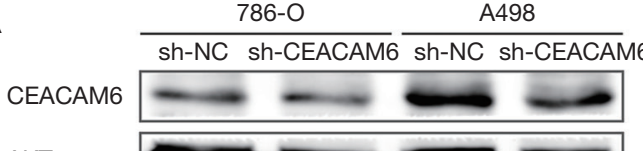

AKT

$\mathrm{p}-\mathrm{AKT}$

ERK

$p-E R K$

C-MYC

MMP-9

Survivin

$\beta$-actin

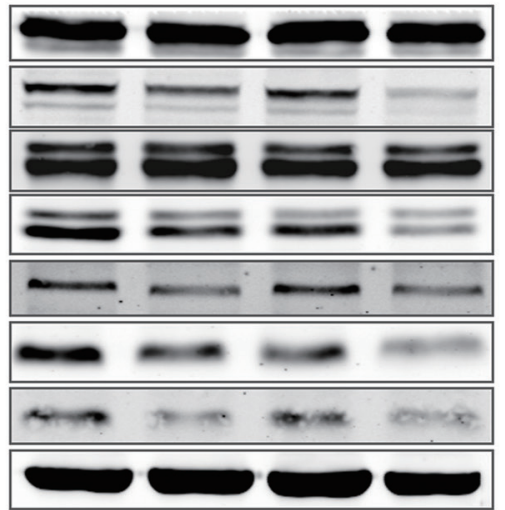

B

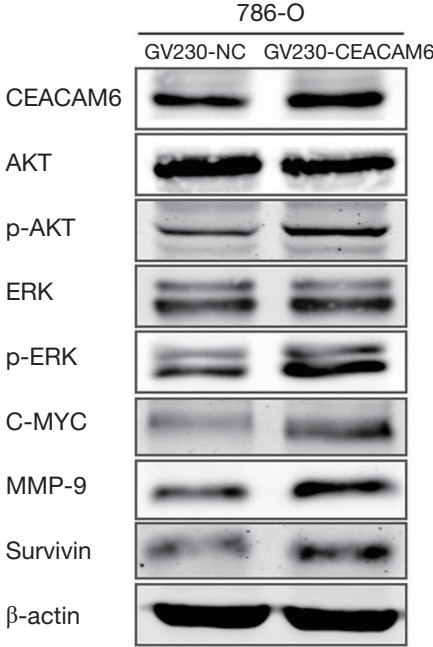

C

$786-\mathrm{O}$
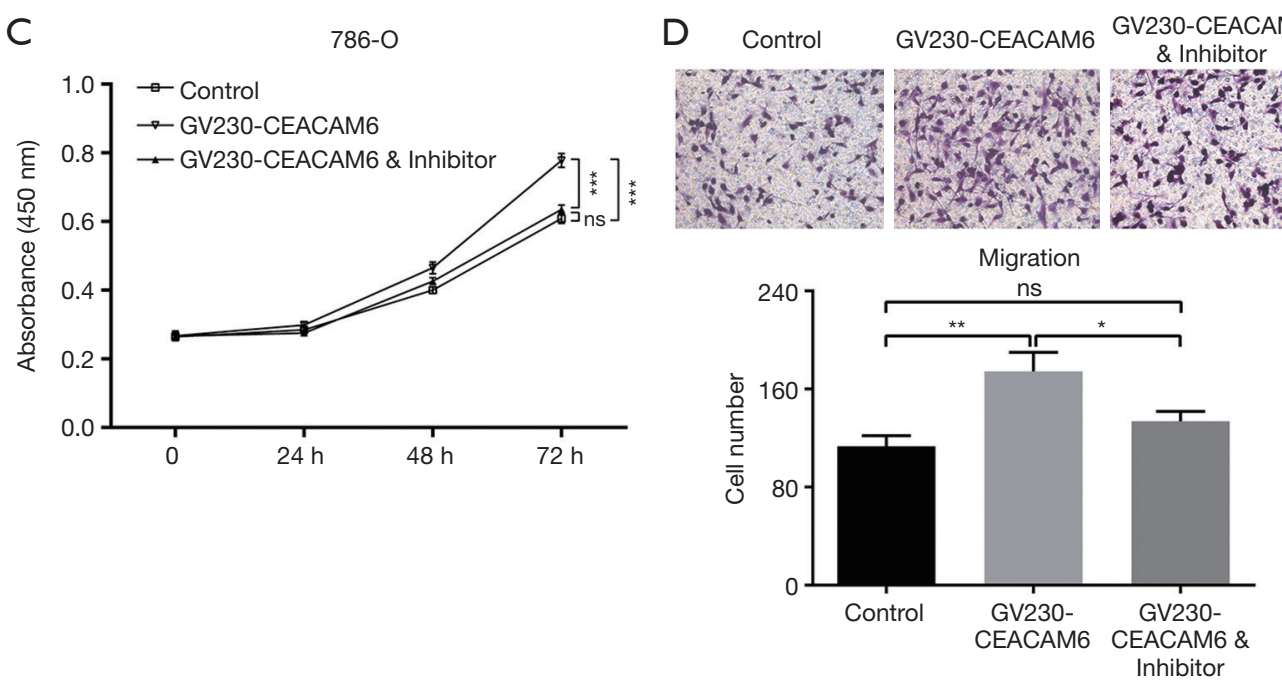

Figure 4 CEACAM6 exerts oncogenic effects in RCC through the ERK/AKT signaling pathway. (A) The protein levels of several pivotal molecules were detected by Western blot following CEACAM6 silencing; (B) the expression of the above molecules following CEACAM6 overexpression. After transfection with GV230-CEACAM6 plasmids, 786-O cells were treated with LY294002 for 24 h. Cell proliferation and migration was detected by CCK-8 assay (C) and transwell assay (D) respectively. Cells were stained by crystal violet, $\times 200$. Data were analyzed by one-way ANOVA. *, $\mathrm{P}<0.05 ;{ }^{* *}, \mathrm{P}<0.01 ;{ }^{* * *}, \mathrm{P}<0.001$. CEACAM6, carcinoembryonic antigen related cell adhesion molecule 6; ns, no significant difference; RCC, renal cell carcinoma.

Overexpression of CEACAM6 has been observed in numerous malignancies, such as pancreatic cancer, gastric cancer, colorectal cancer, cholangiocarcinoma, breast cancer, head and neck cancer, mucinous ovarian neoplasms, leukemias, and RCCs $(17,19,26-32)$. In the present study, we observed overexpression of CEACAM6 in ccRCC tissues by qRT-PCR and immunohistochemical staining, which was further validated in ccRCC cell lines. All of these indicate that overexpression of CEACAM6 may induce the development of ccRCC.

CEACAM6 has been extensively studied as a diagnostic and prognostic marker in a diverse group of malignancies. For example, elevated CEACAM6 levels in atypical ductal hyperplastic lesions can predict the formation of invasive breast cancer (33). High levels of CEACAM6 means shorter overall survival for lung adenocarcinoma patients (34). Previous research revealed an association between CEACAM6 levels and RCC patient 
prognosis (19). Similarly, we found that CEACAM6 expression was negatively correlated with the overall survival of RCC patients, according to survival analysis of the data from the TCGA database. Despite this, the diagnostic and prognostic significance of CEACAM6 in RCC requires further investigation.

By stimulating proliferation, suppressing apoptosis, facilitating migration and invasion, promoting angiogenesis, and inducing drug resistance, CEACAM6 serves as a determinant in the malignant transformation of cells and cancer progression $(11,35)$. To explore the function of CEACAM6 in RCC, CCK-8 and transwell assays were performed in conjunction with the overexpression or silencing of CEACAM6. As expected, excessive CEACAM6 enhanced the capabilities of cell proliferation and migration, while CEACAM6 silencing brought about opposite results. To summarize, CEACAM6 is an oncogene for ccRCC.

Diverse molecular mechanisms are involved in the pathophysiological effects of CEACAM6. In pancreatic adenocarcinomas, overexpression of CEACAM6 facilitates cell invasion through c-Src-mediated increases in MMP-9 activity (27). Moreover, CEACAM6 overexpression induces the phosphorylation activation of AKT and caspase-mediated anoikis resistance, thereby promoting pancreatic cancer metastasis in vivo (36). In gastric cancers, CEACAM6 accelerates metastasis by stimulating the activation of the PI3K/AKT pathway and increasing epithelial-mesenchymal transition (16); CEACAM6 also facilitates angiogenesis through FAK signaling (37). In addition, post-translational modification also plays a critical role in regulating the biological functions of CEACAM6. N-acetylglucosaminyltransferase 5-mediated $\mathrm{N}$-glycosylation of CEACAM6 promotes the aggressive phenotypes of oral squamous cell carcinoma through epidermal growth factor receptor activation triggered signaling cascades (38). According to our research, CEACAM6 silencing suppressed the phosphorylation activation of ERK and AKT and downregulated the expression of C-MYC, Survivin, and MMP-9, thereby restraining the malignant phenotypes of RCC cells. Moreover, PI3K/AKT pathway blockage abrogated CEACAM6 overexpression and increased cell proliferation and migration. More intricate regulatory mechanisms and other pathways beyond ERK/AKT signaling may also be involved and require further investigation.

Considering the excessive expression of CEACAM6 in diverse cancers, depletion of CEACAM6 may be a valid strategy for oncotherapy (39). According to previous studies, miR-29a can suppress the formation and progression of lung adenocarcinomas by attenuating CEACAM6 expression (34). siRNA-mediated CEACAM6 silencing restrains metastasis of pancreatic adenocarcinomas and sensitizes cancer cells to gemcitabine (36,40). A designed humanized anti-CEACAM6 single chain variable fragment can also inhibit the formation of pancreatic cancer (41). Moreover, the integration of CEACAM6-shRNA and yCDglyTK in a recombinant plasmid is an effective gene therapy for pancreatic cancer (42). In addition, 8F5, a monoclonal antibody targeting CEACAM6, increases anoikis, represses tumor growth in vivo, and enhances the therapeutic effects of paclitaxel in lung adenocarcinomas (43). In this study, RNA interference induced loss of function of CEACAM6, which was also effective in suppressing the growth and migration of RCC cells. Collectively, it is promising that targeting CEACAM6 by some specific approaches may represent an effective treatment option for RCC.

In summary, our preliminary study revealed the carcinogenic and prognostic significance of CEACAM6 in ccRCC. It warrants further evaluation for development as a novel therapeutic target for RCC.

\section{Acknowledgments}

Funding: This study was funded by Project of Shanghai Municipal Commission of Health and Family Planning (Grant No. 201740292) and the National Science Foundation of China (31570775 and 81772705).

\section{Footnote}

Conflicts of Interest: The authors have no conflicts of interest to declare.

Ethical Statement: The authors are accountable for all aspects of the work in ensuring that questions related to the accuracy or integrity of any part of the work are appropriately investigated and resolved. This Study was approved by the Ethic Committee of The Affiliated Shanghai Tenth People's Hospital, Nanjing Medical University (2015-35).

\section{References}

1. Moch H, Cubilla AL, Humphrey PA, et al. The 2016 WHO Classification of Tumours of the Urinary System 
and Male Genital Organs-Part A: Renal, Penile, and Testicular Tumours. Eur Urol 2016;70:93-105.

2. Buti S, Bersanelli M, Sikokis A, et al. Chemotherapy in metastatic renal cell carcinoma today? A systematic review. Anticancer Drugs 2013;24:535-54.

3. Van Poppel H, Becker F, Cadeddu JA, et al. Treatment of localised renal cell carcinoma. Eur Urol 2011;60:662-72.

4. Bex A, Jonasch E, Kirkali Z, et al. Integrating surgery with targeted therapies for renal cell carcinoma: current evidence and ongoing trials. Eur Urol 2010;58:819-28.

5. Osorio JC, Motzer RJ, Voss MH. Optimizing Treatment Approaches in Advanced Renal Cancer. Oncology (Williston Park) 2017;31:919-26, 928-30.

6. Posadas EM, Limvorasak S, Figlin RA. Targeted therapies for renal cell carcinoma. Nat Rev Nephrol 2017;13:496-511.

7. Courtney KD, Choueiri TK. Updates on novel therapies for metastatic renal cell carcinoma. Ther Adv Med Oncol 2010;2:209-19.

8. Patil S, Manola J, Elson P, et al. Improvement in overall survival of patients with advanced renal cell carcinoma: prognostic factor trend analysis from an international data set of clinical trials. J Urol 2012;188:2095-100.

9. Hefta SA, Hefta LJ, Lee TD, et al. Carcinoembryonic antigen is anchored to membranes by covalent attachment to a glycosylphosphatidylinositol moiety: identification of the ethanolamine linkage site. Proc Natl Acad Sci U S A 1988;85:4648-52.

10. Benchimol S, Fuks A, Jothy S, et al. Carcinoembryonic antigen, a human tumor marker, functions as an intercellular adhesion molecule. Cell 1989;57:327-34.

11. Beauchemin N, Arabzadeh A. Carcinoembryonic antigenrelated cell adhesion molecules (CEACAMs) in cancer progression and metastasis. Cancer Metastasis Rev 2013;32:643-71.

12. Kuespert K, Pils S, Hauck CR. CEACAMs: their role in physiology and pathophysiology. Curr Opin Cell Biol 2006;18:565-71.

13. Rose JB, Correa-Gallego C, Li Y, et al. The Role of Biliary Carcinoembryonic Antigen-Related Cellular Adhesion Molecule 6 (CEACAM6) as a Biomarker in Cholangiocarcinoma. PLoS One 2016;11:e0150195.

14. Ru GQ, Han Y, Wang W, et al. CEACAM6 is a prognostic biomarker and potential therapeutic target for gastric carcinoma. Oncotarget 2017;8:83673-83.

15. Yan L, Wang Y, Wang ZZ, et al. Cell motility and spreading promoted by CEACAM6 through cyclin D1/ CDK4 in human pancreatic carcinoma. Oncol Rep
2016;35:418-26.

16. Zang M, Zhang B, Zhang Y, et al. CEACAM6 promotes gastric cancer invasion and metastasis by inducing epithelial-mesenchymal transition via PI3K/AKT signaling pathway. PLoS One 2014;9:e112908.

17. Zhang Y, Zang M, Li J, et al. CEACAM6 promotes tumor migration, invasion, and metastasis in gastric cancer. Acta Biochim Biophys Sin (Shanghai) 2014;46:283-90.

18. Kammerer R, Riesenberg R, Weiler C, et al. The tumour suppressor gene CEACAM1 is completely but reversibly downregulated in renal cell carcinoma. J Pathol 2004;204:258-67.

19. Ho TH, Serie DJ, Parasramka M, et al. Differential gene expression profiling of matched primary renal cell carcinoma and metastases reveals upregulation of extracellular matrix genes. Ann Oncol 2017;28:604-10.

20. Chen C, Xue S, Zhang J, et al. DNA-methylationmediated repression of miR-766-3p promotes cell proliferation via targeting SF2 expression in renal cell carcinoma. Int J Cancer 2017;141:1867-78.

21. Guo H, German P, Bai S, et al. The PI3K/AKT Pathway and Renal Cell Carcinoma. J Genet Genomics 2015;42:343-53.

22. Zhai W, Sun Y, Guo C, et al. LncRNA-SARCC suppresses renal cell carcinoma (RCC) progression via altering the androgen receptor(AR)/miRNA-143-3p signals. Cell Death Differ 2017;24:1502-17.

23. Kake S, Usui T, Ohama T, et al. Death-associated protein kinase 3 controls the tumor progression of A549 cells through ERK MAPK/c-Myc signaling. Oncol Rep 2017;37:1100-6.

24. Zhai $W$, Sun $Y$, Jiang $M$, et al. Differential regulation of LncRNA-SARCC suppresses VHL-mutant RCC cell proliferation yet promotes VHL-normal RCC cell proliferation via modulating androgen receptor/ HIF-2alpha/C-MYC axis under hypoxia. Oncogene 2016;35:4866-80.

25. Gold P, Freedman SO. Specific carcinoembryonic antigens of the human digestive system. J Exp Med 1965;122:467-81.

26. Cameron S, de Long LM, Hazar-Rethinam M, et al. Focal overexpression of CEACAM6 contributes to enhanced tumourigenesis in head and neck cancer via suppression of apoptosis. Mol Cancer 2012;11:74.

27. Duxbury MS, Ito H, Benoit E, et al. CEACAM6 is a determinant of pancreatic adenocarcinoma cellular invasiveness. Br J Cancer 2004;91:1384-90.

28. Ieta K, Tanaka F, Utsunomiya T, et al. CEACAM6 gene 
expression in intrahepatic cholangiocarcinoma. $\mathrm{Br} \mathrm{J}$

Cancer 2006;95:532-40.

29. Kim KS, Kim JT, Lee SJ, et al. Overexpression and clinical significance of carcinoembryonic antigen-related cell adhesion molecule 6 in colorectal cancer. Clin Chim Acta 2013;415:12-9.

30. Lasa A, Serrano E, Carricondo M, et al. High expression of CEACAM6 and CEACAM8 mRNA in acute lymphoblastic leukemias. Ann Hematol 2008;87:205-11.

31. Litkouhi B, Litkouhi B, Fleming E, et al. Overexpression of CEACAM6 in borderline and invasive mucinous ovarian neoplasms. Gynecol Oncol 2008;109:234-9.

32. Tsang JY, Kwok YK, Chan KW, et al. Expression and clinical significance of carcinoembryonic antigen-related cell adhesion molecule 6 in breast cancers. Breast Cancer Res Treat 2013;142:311-22.

33. Poola I, Shokrani B, Bhatnagar R, et al. Expression of carcinoembryonic antigen cell adhesion molecule 6 oncoprotein in atypical ductal hyperplastic tissues is associated with the development of invasive breast cancer. Clin Cancer Res 2006;12:4773-83.

34. Han HS, Son SM, Yun J, et al. MicroRNA-29a suppresses the growth, migration, and invasion of lung adenocarcinoma cells by targeting carcinoembryonic antigen-related cell adhesion molecule 6. FEBS Lett 2014;588:3744-50.

35. Rizeq B, Zakaria Z, Ouhtit A. Towards understanding the mechanisms of actions of carcinoembryonic antigenrelated cell adhesion molecule 6 in cancer progression. Cancer Sci 2018;109:33-42.

36. Duxbury MS, Ito H, Zinner MJ, et al. CEACAM6 gene silencing impairs anoikis resistance and in vivo metastatic

Cite this article as: Zhu R, Ge J, Ma J, Zheng J. Carcinoembryonic antigen related cell adhesion molecule 6 promotes the proliferation and migration of renal cancer cells through the ERK/AKT signaling pathway. Transl Androl Urol 2019;8(5):457-466. doi: 10.21037/tau.2019.09.02 ability of pancreatic adenocarcinoma cells. Oncogene 2004;23:465-73.

37. Zang M, Zhang Y, Zhang B, et al. CEACAM6 promotes tumor angiogenesis and vasculogenic mimicry in gastric cancer via FAK signaling. Biochim Biophys Acta 2015;1852:1020-8.

38. Chiang WF, Cheng TM, Chang CC, et al. Carcinoembryonic antigen-related cell adhesion molecule 6 (CEACAM6) promotes EGF receptor signaling of oral squamous cell carcinoma metastasis via the complex N-glycosylation. Oncogene 2018;37:116-27.

39. Johnson B, Mahadevan D. Emerging Role and Targeting of Carcinoembryonic Antigen-related Cell Adhesion Molecule 6 (CEACAM6) in Human Malignancies. Clin Cancer Drugs 2015;2:100-11.

40. Duxbury MS, Ito H, Benoit E, et al. A novel role for carcinoembryonic antigen-related cell adhesion molecule 6 as a determinant of gemcitabine chemoresistance in pancreatic adenocarcinoma cells. Cancer Res 2004;64:3987-93.

41. Riley CJ, Engelhardt KP, Saldanha JW, et al. Design and activity of a murine and humanized anti-CEACAM6 single-chain variable fragment in the treatment of pancreatic cancer. Cancer Res 2009;69:1933-40.

42. Long H, Li Q, Wang Y, et al. Effective combination gene therapy using CEACAM6-shRNA and the fusion suicide gene yCDgly TK for pancreatic carcinoma in vitro. Exp Ther Med 2013;5:155-61.

43. Hong KP, Shin MH, Yoon S, et al. Therapeutic effect of anti CEACAM6 monoclonal antibody against lung adenocarcinoma by enhancing anoikis sensitivity. Biomaterials 2015;67:32-41. 